\title{
Sleep disturbance symptoms and their associations with alexithymia, depression and anxiety
}

\author{
Dionisios Bratis ${ }^{1 *}$, Asimoula Spanopoulou', Silvia Dumitru², Sofia Lagou², Christina Diamandi ${ }^{2}$ Athanasios Tselebis ${ }^{1}$, \\ Georgios Moussas ${ }^{1}$, Athanasios Karkanias', Sotirios Gyftopoulos², Epaminondas Kosmas ${ }^{2}$
}

From $1^{\text {st }}$ International Congress on Neurobiology and Clinical Psychopharmacology and European

Psychiatric Association Conference on Treatment Guidance

Thessaloniki, Greece. 19-22 November 2009

\section{Background}

Several studies indicate that alexithymia and sleep disturbances (especially insomnia) coincide [1]. Aim of the present study is to record the levels of alexithymia, depression and anxiety in a sample of patients with sleep disturbances and to investigate the associations between the above factors.

\section{Materials and methods}

The study included fifty (40 male and 10 female) outpatients who were attended a sleep laboratory of our hospital seeking medical support for symptoms of sleep disturbances. Levels of Alexithymia [2], depression and anxiety [3] [4] were assessed, by using the Toronto Alexithymia Scale (TAS-20), the Beck Depression Inventory (BDI) and the Spielberger Trait Anxiety Inventory (STAI). Age and education level were also recorded.

\section{Results}

Mean BDI score was $10.8 \pm 6.0$, mean STAI score was $45.0 \pm 11.0$ and mean TAS-20 score was $53.8 \pm 14.3$. Mean age and education level were $54.2 \pm 13$ and $10.0 \pm$ 4.1 , respectively. No correlation was observed between the demographic characteristics of the sample and the scores of the clinical measurements (Pearson correlation $\mathrm{p}>0.05$ ). In contrary, a strong positive correlation was presented between TAS-20, BDI and STAI.

\section{Conclusions}

Our preliminary findings confirm the existence of the association between the sleep disturbance symptoms, alexithymia, depression and anxiety. However, some questions remains: are the associations between sleep disturbances and alexithymia caused by depression and/ or anxiety or are independent of them? Further studies are required in order to clarify it.

\section{Author details}

'Dept. of Psychiatry, Sotiria General Hospital of Chest Diseases, Athens, Greece. ${ }^{2}$ Sleep-Disordered Breathing Laboratory of 3rd Dept. of Pulmonary Medicine, Sotiria General Hospital of Chest Diseases, Athens, Greece.

\section{Published: 22 April 2010}

\section{References}

1. Kronholm E, Partonen T, Salminen JK, Mattila AK, Joukamaa M: Alexithymia, Depression and Sleep Disturbance Symptoms. Psychother Psychosom 2008, 77:63-65.

2. Bratis D, Tselebis A, Sikaras C, Moulou A, Giotakis K, Zoumakis E, llias I: Alexithymia and its association with burnout, depression and family support among Greek nursing staff. Hum Resour Health 2009, 7:72.

3. Tselebis A, Gournas G, Tzitzanidou G, Panagiotou A, llias I: Anxiety and depression in Greek nursing and medical personnel. Psychol Rep 2006, 99(1):93-6.

\section{doi:10.1186/1744-859X-9-S1-S163}

Cite this article as: Bratis et al:: Sleep disturbance symptoms and their associations with alexithymia, depression and anxiety. Annals of General Psychiatry 2010 9(Suppl 1):S163. 